\title{
Parameterized Algorithm for Eternal Vertex Cover
}

\author{
Fedor V. Fomin* $\quad$ Serge Gaspers ${ }^{\dagger} \quad$ Petr A. Golovach* Dieter Kratsch $^{\ddagger}$ \\ Saket Saurabh*
}

September 4, 2009

\begin{abstract}
In this paper we initiate the study of a "dynamic" variant of the classical VerTex Cover problem, the ETERNAL VERTEX COVER problem, from parameterized algorithmic perspective. Klostermeyer and Mynhardt introduced the Eternal Vertex Cover problem, which consists in placing a minimum number of guards on the vertices of a graph such that these guards can protect the graph from any sequence of attacks on its edges. In response to an attack, each guard is allowed either to stay in his vertex, or to move to a neighboring vertex. However, at least one guard has to fix the attacked edge by moving along it. The other guards may move to reconfigure and prepare for the next attack. Thus at every step the vertices occupied by guards form a vertex cover. We show that the problem admits a kernel of size $4^{k}(k+1)+2 k$, which shows that the problem is fixed parameter tractable when parameterized by the number of available guards $k$. Finally, we also provide an algorithm with running time $\mathcal{O}\left(2^{\mathcal{O}\left(k^{2}\right)}+(1.2738)^{k} m+n\right)$ for Eternal Vertex Cover, where $n$ is the number of vertices and $m$ the number of edges of the input graph. In passing we also observe that Eternal Vertex Cover is NP-hard but that there is a polynomial time 2-approximation algorithm.
\end{abstract}

\section{Introduction}

In the classical Vertex COVER problem we are given a graph $G=(V, E)$ and a positive integer $k$ as input and the objective is to decide whether there exists a subset $C$ of the vertex set $V$ of size at most $k$ such that it contains at least one vertex from each of the edges in the graph. Klostermeyer and Mynhardt [7] introduced a dynamic variant of the VERTEX Cover problem, the Eternal Vertex Cover problem. In the Eternal Vertex Cover problem, the input is a graph $G=(V, E)$ on $n$ vertices and $m$ edges. Guards are placed on the vertices of the graph in order to protect it from an infinite sequence (which is not known to the guards in advance) of attacks on the edges of the graph. In each round, one edge $e \in E$ is attacked, and each guard moves from the vertex it is occupying to a vertex in the closed neighborhood of that vertex, such that at least one guard moves along $e$. The minimum number of guards which can protect all the edges of $G$ is called the eternal vertex cover number of $G$ and is denoted by $\operatorname{evcn}(G)$. The Eternal Vertex Cover problem is to compute $\operatorname{evcn}(G)$ for a graph $G$, and the parameterized version of the problem is to decide whether $\operatorname{evcn}(G) \leq k$ for a graph $G$ and a parameter $k$.

In Parameterized Complexity, one asks if for an instance of size $n$ and a parameter $k$, a problem can be solved in time $f(k) n^{\mathcal{O}(1)}$ where $f$ is an arbitrary computable function independent of $n$. Problems that can be solved in that time are said to be fixed parameter tractable,

\footnotetext{
${ }^{*}$ Department of Informatics, University of Bergen, N-5020 Bergen, Norway.

${ }^{\dagger}$ LIRMM - University of Montpellier 2, CNRS, 34392 Montpellier, France.

${ }^{\ddagger}$ Laboratoire d’Informatique Théorique et Appliquée, Université Paul Verlaine - Metz, 57045 Metz Cedex 01, France.
} 
and the corresponding complexity class is called FPT. We refer to the books of Downey and Fellows [5] and Niedermeier [11] for an introduction to the area.

The Vertex COVER problem is one of the best studied problems in the realm of parameterized complexity and a lot of research has been done on it. The current fastest algorithm for Vertex COver runs in time $1.2738^{k} n^{\mathcal{O}(1)}$ [2]. As observed by many, Vertex Cover could be considered the "Drosophila" of fixed-parameter algorithmics [5, 6,11$]$. The literature on parameterized complexity is full of variants of Vertex Cover like Connected Vertex Cover [6,10], Capacitated Vertex Cover [4,6], and Partial Vertex Cover [8,12]. In this paper we show that its dynamic variant, namely $p$-Eternal VERTEX COVER, is also fixed parameter tractable.

The paper is organized as follows. In Section 2 we give some basic definitions and observe that the parameterized complexity of ETERNAL VERTEX COVER is the same when parameterized by the number of available guards and when parameterized by the number of vertices that are occupied by guards (a vertex may be occupied by more than one guard). Section 3 deals with exact and parameterized algorithms for Eternal VerTex COVER. We start with an $2^{\mathcal{O}(n)}$ time algorithm computing the eternal vertex cover number of an $n$-vertex graph. Then we prove FPT membership by providing a $k^{\prime}=4^{k}(k+1)+2 k$ kernel, which combined with the exact algorithm, leads to a time $2^{\mathcal{O}\left(k^{\prime}\right)} \cdot n^{\mathcal{O}(1)}$ algorithm. We then improve on this algorithm and give an algorithm with running time $\mathcal{O}\left(2^{\mathcal{O}\left(k^{2}\right)}+1.2738^{k} m+n\right)$. We conclude with some remarks in Section 4.

\section{Preliminaries}

All graphs in this paper are finite, undirected and without multiple edges and loops. For notation and terminology not defined in this paper we refer to [3]. Let $G=(V, E)$ be a graph. A set $S \subseteq V$ is a vertex cover of $G$, if for every edge of $E$ at least one endpoint is in $S$.

In view of the study of the ETERNAL VERTEX COVER problem from the parameterized complexity point of view in Section 3, we use the following easy lemma from [7], which shows that the parameterized complexity of the problem remains the same when it is parameterized by the number of guards available to protect the graph or the number of vertices which may be occupied by guards. Let $\mathbf{v c}(G)$ denote the minimum size of a vertex cover of $G$, and $\operatorname{egvn}(G)$ denote the minimum number of vertices occupied by guards in an initial configuration of guards that can eternally protect the edges of $G$.

Lemma $1[7]$ For any graph $G, \operatorname{vc}(G) \leq \operatorname{egvn}(G) \leq \operatorname{evcn}(G) \leq 2 \mathbf{v c}(G)$.

Let us remark that the three inequalities of Lemma 1 are tight: $\operatorname{vc}\left(P_{2}\right)=\operatorname{egvn}\left(P_{2}\right)=$ $\operatorname{evcn}\left(P_{2}\right)=1$ and $\operatorname{evcn}\left(P_{3}\right)=2 \operatorname{egvn}(G)=2 \mathbf{v c}\left(P_{3}\right)=2$, where $P_{n}$ denotes a path on $n$ vertices.

\section{$3 \quad$ FPT Algorithm for Eternal Vertex Cover}

We start by observing that Eternal Vertex Cover is indeed $N P$-hard by giving a simple reduction from VERTEX COVER. Then we obtain an exact algorithm running in time $2^{\mathcal{O}(n)}$ to find the eternal vertex cover number of a graph $G=(V, E)$ on $n$ vertices and $m$ edges. A "trimmed variant" of this algorithm is crucially used in obtaining the faster fixed parameter tractable algorithm for ETERnAL VerTex COVER. Finally, we give our kernel and the algorithm for Eternal Vertex Cover.

Proposition 1 It is NP-hard to decide whether $k$ guards can protect all the edges of a graph. 
Proof: We reduce from the Vertex Cover problem. Recall that it asks about the existence of a vertex cover of the size at most $k$ in a graph $G$. Assume that $k<|V(G)|-1$, since VerTex Cover obviously remains $N P$-complete with this additional condition. We add a new universal vertex $u$ and join it with all vertices of $G$ by edges. Then a vertex $v$ is added and joined with $u$. Denote the obtained graph by $H$. We claim that $G$ has a vertex cover of size at most $k$ if and only if $k+2$ guards can protect all the edges of $H$. The correctness of the reduction is easy to see.

The following lemma provides a simple result on the approximability of the problem.

Lemma 2 There is a 2-approximation algorithm for the ETERNAL VERTEX COVER problem with running time $\mathcal{O}(\sqrt{n} \cdot m)$, where $n$ is the number of vertices and $m$ is the number of edges of the input graph.

Proof: Let $M$ be a maximum matching of size $k$ in a graph $G$. Then the size of a minimum vertex cover of $G$ is at least $k$, and thus $\operatorname{evcn}(G) \geq k$. By placing guards on the endpoints of the edges in $M$, we use $2 k$ guards. We claim that the guards occupying the endpoints of the edges of a maximum matching in $G$ can always protect $G$. Indeed, the guards' strategy is as follows. Consider an attack on an edge $x y$. If both $x$ and $y$ are occupied by a guard, then these two guards swap their positions and the other guards do not move. Thus we are exactly in the same position as before the attack. If exactly one endpoint of $x y$, say $x$, is occupied by a guard, then there is an edge $x z \in M$, and $y$ is not incident to an edge in $M$. Therefore, $M^{\prime}=M \backslash\{x z\} \cup\{x y\}$ is also a matching of size $k$. We move a guard from $x$ to $y$, and a guard from $z$ to $x$. Thus the guards occupy the endpoints of the maximum matching $M^{\prime}$.

Finally, a maximum matching in a graph can be found in time $\mathcal{O}(\sqrt{n} \cdot m)[9]$.

\subsection{Exact Algorithm for Eternal Vertex Cover}

In this section we give an exact algorithm running in time $2^{\mathcal{O}(n)}$ to find the eternal vertex cover number of a graph $G=(V, E)$ on $n$ vertices and $m$ edges.

For every $p, 1 \leq p \leq n$, we want to decide if $p$ guards can protect the graph. To solve the problem we construct a configuration multi-graph $\mathcal{G}=(\mathcal{V}, \mathcal{E})$ from $G$ as follows. Let $V(G)=\left\{v_{1}, \ldots, v_{n}\right\}$. A vertex $\mathcal{I} \in \mathcal{V}$ is a vector $\left(\left(v_{1}, p_{1}\right), \ldots,\left(v_{n}, p_{n}\right)\right)$ of pairs $\left(v_{i}, p_{i}\right)$ where $p_{i} \in\{0, \ldots, p\}$ such that $\sum_{i=1}^{n} p_{i}=p$. A pair $\left(v_{i}, p_{i}\right)$ represents the situation when there are $p_{i}$ guards on vertex $v_{i}$.

Now for every edge $e$ of $G$, we add an edge from vertex $\mathcal{I}$ to vertex $\mathcal{J}$ (we call it e-edge) if by the attack on $e$, the guards can move from the position corresponding to $\mathcal{I}$ to the position corresponding to $\mathcal{J}$, in a way that at least one of the guards slides along $e$. Notice that it is possible that $\mathcal{I}=\mathcal{J}$, i.e. $\mathcal{G}$ can have loops. Note also that if by the attack on $e$, the guards can move from the position corresponding to $\mathcal{I}$ to the position corresponding to $\mathcal{J}$, then the guards can move from the position corresponding to $\mathcal{J}$ to the position corresponding to $\mathcal{I}$ to counter the attack on $e$.

Lemma 3 Deciding whether $(\mathcal{I}, \mathcal{J})$ is an e-edge can be done in time $n^{\mathcal{O}(1)}$.

Proof: To decide if there is an $e$-edge from $\mathcal{I}$ to $\mathcal{J}$, we solve a flow problem on an auxiliary directed graph $H$ whose edges have capacities and costs. For the construction of $H$, make two copies $V_{1}$ and $V_{2}$ of the vertex set of $G$ and construct a bipartite graph by connecting every vertex $v \in V_{1}$ to the vertices of $V_{2}$ from its closed neighborhood. We give cost 1 to all the edges constructed so far, except the two copies of $e$, which obtain cost 0 . The capacities of the edges that have been constructed by now are set equal to $p$ (or unrestricted capacity). We also add two vertices $s$ and $t$, and connect $s$ to $V_{1}$ and $V_{2}$ to $t$. Then, for every edge $s v_{i}, v_{i} \in V_{1}$ we put 
its capacity equal to the number of guards $p_{i}$ located on $v_{i}$ in configuration $\mathcal{I}$ and its cost equal to 0 . Correspondingly, the capacity of edge $u t, u \in V_{2}$, is the number of guards on $u$ in $\mathcal{J}$ and its cost is 0 . Now the question if there is an $e$-edge from $\mathcal{I}$ to $\mathcal{J}$ can be answered by finding a minimum cost flow from $s$ to $t$ in the auxiliary graph, which is solvable in polynomial (in $n$ ) time. Namely, $(\mathcal{I}, \mathcal{J})$ is an $e$-edge if and only if there is a $s-t$ flow in $H$ of value $p$ with cost at most $p-1$ (i.e. at least one of the copies of $e$ has non-zero flow).

We call a vertex $\mathcal{I}$ safe if for any edge $e \in E$ there is an $e$-edge incident to $\mathcal{I}$ in $\mathcal{G}$. We recursively check whether a vertex of $\mathcal{G}$ is safe or not and if it is not then we remove the vertex. We do this until all vertices of $\mathcal{G}$ are safe. Observe that we can check whether a vertex is safe or not in time proportional to its degree, and check whether every vertex of $\mathcal{G}$ is safe or not in time $\mathcal{O}(|\mathcal{E}|)$. Furthermore, the number of times we have to check whether the graph is safe or not is at most $|\mathcal{V}|$, as every time we delete at least one vertex from the graph $\mathcal{G}$. We arrive at the following lemma.

Lemma $4 A$ graph $G$ has an eternal vertex cover of size $p$ if and only if $\mathcal{G}$ is non-empty after recursively removing all the unsafe vertices of $\mathcal{G}$. It can be checked in time $\mathcal{O}(|\mathcal{E}| \cdot|\mathcal{V}|)$ whether $G$ is safe.

Theorem 1 Given a graph $G=(V, E)$ on $n$ vertices, it is possible to compute $\operatorname{evcn}(G)$ in time $2^{\mathcal{O}(n)}$.

Proof: To compute $\operatorname{evcn}(G)$, for every $p$ from 1 to $n$, we compute the auxiliary graph $\mathcal{G}=$ $(\mathcal{V}, \mathcal{E})$. The number of vertices in $\mathcal{G}$ is $|\mathcal{V}| \leq\left(\begin{array}{c}n+p-1 \\ p\end{array}\right) \leq 2^{2 n}$ and the number of edges $|\mathcal{E}| \leq$ $|E| \frac{|\mathcal{V}|(|\mathcal{V}|+1)}{2}$. By Lemma 3, such a graph can be constructed in $|\mathcal{E}| \cdot n^{\mathcal{O}(1)}$ steps. By Lemma 4 , given a graph $\mathcal{G}$, checking if $G$ is safe and thus $\operatorname{evcn}(G) \leq p$, can be performed in time $\mathcal{O}(|\mathcal{E}|$. $|\mathcal{V}|)=\mathcal{O}\left(64^{n} \cdot n^{\mathcal{O}(1)}\right)=2^{\mathcal{O}(n)}$

\subsection{An FPT Algorithm for Eternal Vertex Cover}

Let us start by defining the notion of kernelization. Recall that a parameterized problem $Q$ is formally a subset of $\Sigma^{*} \times \mathbb{N}$ for some finite alphabet $\Sigma$, and an instance of a parameterized problem is the pair $(x, k) \in \Sigma^{*} \times \mathbb{N}$, where $k$ is called the parameter.

Definition 1 A kernelization algorithm for a parameterized problem $Q \subseteq \Sigma^{*} \times \mathbb{N}$ is an algorithm that, given $(x, k) \in \Sigma^{*} \times \mathbb{N}$, outputs in time polynomial in $|x|+k$ a pair $\left(x^{\prime}, k^{\prime}\right) \in \Sigma \times \mathbb{N}$ such that

- for all $(x, k) \in Q$ if and only if $\left(x^{\prime}, k^{\prime}\right) \in Q$,

- there is a computable function $g$ with the property that $\left|x^{\prime}\right|+k^{\prime} \leq g(k)$ for any instance $(x, k)$.

If such a kernelization algorithm exists for a parameterized problem $Q$, we say that $Q$ has a kernel of size $g$. It is well known (see, e.g. [11]) that a decidable parameterized problem is in FPT if and only if it admits a kernel, that is a reduction of the problem and its parameter to an instance of size bounded by a function of $k$.

Our kernelization algorithm relies on the fact that at any step the positions occupied by the guards must form a vertex cover of size at most $k$ of the graph $G$. It works as follows:

1. Apply a factor 2 approximation algorithm on $G$ (Lemma 2), and obtain a minimal vertex cover $S$. 
2. If $|S|>2 k$, then return that $G$ does not have an eternal vertex cover of size at most $k$.

3. Else, for every subset $T \subseteq S$ we define $\mathcal{S}(T)$ as the set of vertices of $I=V \backslash S$ ( $I$ is an independent set) such that for every $u \in \mathcal{S}(T), N(u)=T$. If any such set $\mathcal{S}(T)$ contains more than $k+1$ vertices then we remove all the vertices except some $k+1$ of them. Let $G(S)$ be the graph obtained after having considered all subsets $T \subseteq S$.

This concludes the description of the kernelization algorithm. Next we show the correctness of the algorithm.

Lemma 5 Let $G$ be a graph on $n$ vertices and $m$ edges. Then $G$ has an eternal vertex cover of size at most $k$ if and only if $G(S)$ has an eternal vertex cover of size at most $k$.

Proof: The idea of the proof is that for a given subset $T \subseteq S$, any pair of vertices in $\mathcal{S}(T)$ is indistinguishable. Also we use the fact that if $|\mathcal{S}(T)| \geq k+1$ then at least one vertex of $\mathcal{S}(T)$ is not occupied by guards.

Denote by $T_{1}, \ldots, T_{r}$ subsets of $S$ such that $\left|\mathcal{S}\left(T_{j}\right)\right|>k+1$ in $G$. Let $U_{j}=\mathcal{S}\left(T_{j}\right)$ in $G$, and let $W_{j}=U_{j} \cap V(G(S))$ for $j \in\{1, \ldots, r\}$.

Suppose that $G$ has an eternal vertex cover of size at most $k$. Denote by $\mathcal{H}$ the graph obtained from the configuration graph $\mathcal{G}$ of $G$ for $k$ guards after removing all unsafe vertices. We choose a vertex $w_{j}$ in each set $W_{j}$. For each vertex $\mathcal{I} \in V(\mathcal{H})$, we construct the vertex $\mathcal{J}(\mathcal{I})$ in the configuration graph for $G(S)$ in the following way. Recall that the vertex $\mathcal{I}$ is a vector $\left(\left(v_{1}, p_{1}\right), \ldots,\left(v_{n}, p_{n}\right)\right)$ where $\left\{v_{1}, \ldots, v_{n}\right\}=V(G)$ and $p_{i}$ is the number of guards in $v_{i}$. To define $\mathcal{J}(\mathcal{I})$, we have to define the number of guards $q_{i}$ in each $v_{i} \in V(G(S)$ ). For any $v_{i} \in V(G(S)) \backslash\left\{w_{1}, \ldots, w_{r}\right\}$, let $q_{i}=p_{i}$. For $v_{i}=w_{j}$, the number of guards in it equals the total number of guards in $v_{i}$ and on vertices of $U_{j} \backslash W_{j}$ for $\mathcal{I}$. Denote by $\mathcal{H}^{\prime}$ the subgraph of the configuration graph for $G(S)$ induced by the set of different vertices $\mathcal{J}(\mathcal{I})$ for all $\mathcal{I} \in V(\mathcal{H})$. Let $e \in E(G(S))$ and let $\mathcal{J}$ be a vertex of $\mathcal{H}^{\prime}$. Suppose that $\mathcal{J}=\mathcal{J}(\mathcal{I})$ for $\mathcal{I} \in \mathcal{H}$. Since $\mathcal{I}$ is a safe vertex, there is a $\mathcal{I}^{\prime} \in V(\mathcal{H})$ such that $\left(\mathcal{I}, \mathcal{I}^{\prime}\right)$ is an $e$-edge. Observe that $\left(\mathcal{J}(\mathcal{I}), \mathcal{J}\left(\mathcal{I}^{\prime}\right)\right)$ is an $e$-edge in the configuration graph for $G(S)$. Hence all vertices of $\mathcal{H}^{\prime}$ are safe, and therefore $\operatorname{evcn}(G(S)) \leq k$.

Assume now that $G(S)$ has an eternal vertex cover of size at most $k$. Denote by $\mathcal{H}^{\prime}$ the graph obtained from the configuration graph of $G(S)$ for $k$ guards after removing all unsafe vertices. For each vertex $\mathcal{J} \in \mathcal{H}^{\prime}$, we construct a collection of vertices of $\mathcal{G}$ by considering all possible injective mappings $h_{j}: W_{j} \rightarrow U_{j}$ for $j \in\{1, \ldots, r\}$. Let $q_{i}$ be the number of guards in $v_{i} \in V(G(S))$ in the configuration corresponding to $\mathcal{J}$. We define the vertex $\mathcal{I}=$ $\left(\left(v_{1}, p_{1}\right), \ldots,\left(v_{n}, p_{n}\right)\right) \in \mathcal{I}$ for mappings $h_{1}, \ldots, h_{r}$ in the following way. For each $v_{i} \in V(G) \backslash$ $\left(U_{1} \cup \cdots \cup U_{r}\right)$, we set $p_{i}=q_{i}$. For any $v_{i} \in W_{j}$, the number of guards in $h_{j}\left(v_{i}\right)$ is $q_{i}$. For remaining vertices of $U_{j}$, the number of guards is equal to 0 . Denote by $\mathcal{H}$ the subgraph of the configuration graph for $G$ induced by all constructed vertices $I$ for all possible collections of mappings $h_{1}, \ldots, h_{r}$. We claim that all vertices $\mathcal{H}$ are safe, and hence $\operatorname{evcn}(G) \leq k$.

To prove the claim, we consider an edge $e=u v \in E(G)$ and $\mathcal{I} \in V(\mathcal{H})$. It is necessary to prove that there is $\mathcal{I}^{\prime} \in V(\mathcal{H})$ such that $\left(\mathcal{I}, \mathcal{I}^{\prime}\right)$ is an $e$-edge in $\mathcal{H}$. Assume that $\mathcal{I}$ is constructed from $\mathcal{J} \in \mathcal{H}^{\prime}$ by the use of mappings $h_{1}, \ldots, h_{r}$. We consider two cases:

1. $u, v \in V(G)$ but $u, v \notin\left(U_{1} \backslash h_{1}\left(W_{1}\right)\right) \cup \ldots \cup\left(U_{r} \backslash h_{r}\left(W_{r}\right)\right)$. Since $J$ is a safe vertex, there is another vertex $\mathcal{J}^{\prime}$ in $\mathcal{H}^{\prime}$ such that $\left(\mathcal{J}, \mathcal{J}^{\prime}\right)$ is an $e$-edge in $\mathcal{H}^{\prime}$. Let $\mathcal{I}^{\prime}$ be the vertex of $\mathcal{H}$ constructed by the use of same mappings $h_{1}, \ldots, h_{r}$. Then $\left(\mathcal{I}, \mathcal{I}^{\prime}\right)$ is an e-edge in $\mathcal{H}$.

2. $u \in S$ and $v \in U_{j} \backslash h_{j}\left(W_{j}\right)$ for some $j \in\{1, \ldots, r\}$. Since $\left|W_{j}\right|=k+1$, there is a vertex $w \in W_{j}$ such that $w$ is not occupied by guards in the position corresponding to $J$. Replace the mappings $h_{1}, \ldots, h_{r}$ by mappings $h_{1}^{\prime}, \ldots, h_{r}^{\prime}$ such that $h_{i}^{\prime}=h_{i}$ if $i \neq j$, 
and $h_{j}^{\prime}(x)=\left\{\begin{array}{ll}h_{j}(x), & \text { if } x \neq w, \\ v, & \text { if } x=w .\end{array}\right.$ It remains to observe that it can be assumed that $\mathcal{I}$ is constructed from $J$ by use of the mappings $h_{1}^{\prime}, \ldots, h_{r}^{\prime}$ instead $h_{1}, \ldots, h_{r}$, and since $v \in h_{j}^{\prime}\left(W_{j}\right)$, this case is reduced to Case 1 .

This immediately implies the following theorem.

Theorem 2 Given a graph $G$ on $n$ vertices and $m$ edges and an integer $k$, in $\mathcal{O}(m+n)$ time we can obtain a kernel for ETERNAL VERTEX COVER of size at most $k^{\prime}=4^{k}(k+1)+2 k$.

We can easily obtain an FPT algorithm by combining Theorems 2 and 1 . This will give an FPT algorithm of running time $64^{k^{\prime}} n^{\mathcal{O}(1)}$. In what follows, we improve upon this running time with the following algorithm.

Step 1: Find a vertex cover $S$ of size at most $k$ of $G$ using the fastest parameterized algorithm for $k$-Vertex Cover [2].

Step 2: For every subset $T \subseteq S$, we define $\mathcal{S}(T)$ as the set of vertices of $I=V \backslash S$ ( $I$ is an independent set) such that for every $u \in \mathcal{S}(T), N(u)=T$. Observe that this partitions the vertices of the independent set $I$. If any set $\mathcal{S}(T)$ contains more than $k+1$ vertices then we remove all the vertices except some $k+1$ of them. Let $G(S)$ be the graph obtained by this procedure.

Step 3: Check whether $G(S)$ has an eternal vertex cover of size at most $k$ using the algorithm described in the previous section. If for $G(S)$ there is an eternal vertex cover of size at most $k$ then return YES else return No.

The correctness of the above described algorithm is not hard to see. For the time complexity observe that the size of $S$ is at most $k$ and that the size of $G(S)$ is bounded by $k^{\prime}=2^{k}(k+1)+k$. Using the fact that the number of guards $k$ is considerably smaller than $k^{\prime}$, we can improve the running time bound for our exact algorithm. The configuration graph $\mathcal{G}_{S}$ for $G(S)$ and $k$ guards has at most

$$
\left(\begin{array}{c}
2^{k}(k+1)+2 k-1 \\
k
\end{array}\right) \leq 2^{\mathcal{O}\left(k^{2}\right)}
$$

vertices. Hence the algorithm has running time $2^{\mathcal{O}\left(k^{2}\right)}$. This brings us to the following theorem.

Theorem 3 Let $G$ be a graph on $n$ vertices and $m$ edges and $k$ be a positive integer. It is possible to check whether $G$ has an eternal vertex cover of size at most $k$ in time $\mathcal{O}\left(2^{O\left(k^{2}\right)}+\right.$ $\left.(1.2738)^{k} m+n\right)$.

\section{Concluding Remarks and Open Problems}

In this paper we initiated the parameterized study of the ETERnAL VeRTEx COVER problem. We showed that the problem is fixed parameter tractable by giving a kernel of size $4^{k}(k+1)+2 k$. There are still many interesting questions regarding the problem which remain unanswered. Does there exist a polynomial size kernel for the problem? Or can we prove that no polynomial kernel exists for the problem, using the recently developed methodology to show non-existence of polynomial kernels [1]. Can we find an $2^{\mathcal{O}(k)} n^{\mathcal{O}(1)}$ algorithm for the problem? From the classical complexity perspective the challenging question is if the ETERNAL VERTEx Cover problem is in $N P$ ? 


\section{References}

[1] H. L. Bodlaender,R. G. Downey, M. R. Fellows and D. Hermelin, On Problems without Polynomial Kernels (Extended Abstract), Proceedings of ICALP 2008, Springer, LNCS 5125 (2008), 563-574.

[2] J. Chen, I. A. Kanj, and G. Xia, Improved Parameterized Upper Bounds for Vertex Cover, Proceedings of MFCS 2006, Springer, LNCS 4162 (2006), 238-249.

[3] R. Diestel. Graph Theory, Second edition, Graduate Texts in Mathematics 173, Springer, 2000 .

[4] M. Dom, D. Lokshtanov, S. Saurabh and Y. Villanger, Capacitated Domination and Covering: A Parameterized Perspective, Proceedings of IWPEC 2008, Springer, LNCS 5018 (2008), 78-90.

[5] R. G. Downey and M. R. Fellows, Parameterized Complexity, Springer-Verlag, 1999.

[6] J Guo, R. Niedermeier and S. Wernicke, Parameterized Complexity of Vertex Cover Variants, Theory Comput. Syst. 41(3) (2007), 501-520.

[7] W. F. Klostermeyer and C. M. Mynhardt, Eternal Protection in Graphs, To appear in Australasian Journal of Combinatorics (2009).

[8] J. Kneis, A. Langer and P. Rossmanith, Improved Upper Bounds for Partial Vertex Cover, Proceedings of WG 2008, Springer, LNCS 5344 (2008), 240-251.

[9] S. Micali and V. V. Vazirani, An $O(\sqrt{|V|} \cdot|E|)$ Algorithm for Finding Maximum Matching in General Graphs, in FOCS 1980, pp. 17-27.

[10] D. Mölle, S. Richter and P. Rossmanith, Enumerate and Expand: Improved Algorithms for Connected Vertex Cover and Tree Cover, Theor. Comput. Syst. 43(2) (2008), 234-253.

[11] R. Niedermeier, Invitation to fixed-parameter algorithms, Oxford Lecture Series in Mathematics and Its Applications 31, Oxford University Press, USA, 2002.

[12] V. Raman and S. Saurabh, Short Cycles Make W-hard Problems Hard: FPT Algorithms for W-hard Problems in Graphs with no Short Cycles, Algorithmica 52(2) (2008), 203-225. 